
\section{$\overbrace{\substack{\text { CHEST } \\ \text { CLNIC }}}$} Children's Hospital, Melbourne, Victoria, Australia

${ }^{2}$ Department of General Medicine, Royal Children's Hospital, Melbourne, Australia ${ }^{3}$ Department of Paediatrics, The University of Melbourne, Melbourne, Victoria, Australia ${ }^{4}$ Murdoch Children's Research Institute, Parkville, Victoria, Australia

${ }^{5}$ Department of Immunology, Royal Children's Hospital, Melbourne, Australia

\section{Correspondence to} Dr Shivanthan Shanthikumar, Department of Respiratory Medicine, Royal Children's Hospital, 50 Flemington Road, Parkville, Melbourne, VIC, 3052, Australia; shivanthan. shanthikumar@rch.org.au

Received 18 April 2016 Revised 12 July 2016 Accepted 17 July 2016 Published Online First 8 August 2016

\title{
Community-acquired pneumonia in children: what to do when there is no response to standard empirical treatment?
}

\author{
Shivanthan Shanthikumar, ${ }^{1,3,4}$ Amelia Clifford, ${ }^{2}$ John Massie, ${ }^{1,3,4}$ Theresa Cole, ${ }^{4,5}$ \\ Andrew Steer, ${ }^{2,3,4}$ Michael Marks, ${ }^{2,3}$ Amanda Gwee ${ }^{2,3,4}$
}

\section{SS (REGISTRAR) AND AC (RESIDENT)}

A 5-year-old female presents to a tertiary paediatric hospital in Australia with a 2-day history of fever, vomiting and abdominal pain. Her past history consists of three episodes of otitis media in the preceding 12 months, all resulting in tympanic membrane perforation. Her parents report an 'innocent heart murmur' diagnosed by a paediatric cardiologist. There is no history of travel or animal exposure. With regard to family history, she is the child of non-consanguineous Caucasian parents, and her father has Crohn's disease. On examination, she is febrile $\left(40.0^{\circ} \mathrm{C}\right)$ and tachypnoeic $(45$ breaths $/ \mathrm{min})$ with normal oxygen saturations (99\% room air). Bronchial breath sounds are auscultated posteriorly in the left lower zone. She has a 3/6 systolic murmur heard at the apex and lower left sternal edge with no radiation. Her first full blood examination showed a neutrophilia $\left(13.9 \times 10^{9} / \mathrm{L}\right.$, normal $\left.<8.5 \times 10^{9} / \mathrm{L}\right)$ and mild lymphopenia $\left(1.9 \times 10^{9} / \mathrm{L}\right.$, normal $\left.>2.0 \times 10^{9} / \mathrm{L}\right)$ which resolved. Her C-reactive protein (CRP) is elevated at $157 \mathrm{mg} / \mathrm{L}$ (normal $<8 \mathrm{mg} / \mathrm{L}$ ). A chest radiograph (CXR) shows left lower lobe consolidation with an irregular area of radiolucency suggestive of an evolving abscess (see figure $1 \mathrm{~A}$ ). The patient is commenced on intravenous flucloxacillin $(50 \mathrm{mg} / \mathrm{kg}, 6$ hourly) and ceftriaxone $(50 \mathrm{mg} / \mathrm{kg}, 12$ hourly). Despite this, she continues to spike high-grade fevers $\left(>39.0^{\circ} \mathrm{C}\right)$. Blood cultures on two occasions are negative.

\section{AS (GENERAL PAEDIATRICIAN AND PAEDIATRIC INFECTIOUS DISEASES PHYSICIAN)}

The initial presentation with fever and abdominal pain is not uncommon in patients with pneumonia. Tachypnoea is a sensitive and specific sign of pneumonia, and CXR is the most appropriate initial diagnostic investigation. ${ }^{1}$ The presence of fever, elevated inflammatory markers and in particular the CXR findings suggest a complicated pneumonia, possibly a lung abscess. Given the suspicion of a lung abscess, it is appropriate to commence broader antibiotic coverage rather than start with intravenous benzylpenicillin (recommended firstline intravenous antibiotic for those patients with community-acquired pneumonia (CAP) who are admitted to hospital). ${ }^{1} \quad{ }^{2}$ For primary lung abscesses, it is important to cover Staphylococcus aureus, Streptococcus pneumoniae and other streptococcal species. ${ }^{3}$ There is debate regarding the optimal empiric antibiotic regimen; institutional guidelines vary, and many recommend flucloxacillin and ceftriaxone. ${ }^{3}$ The acute presentation is not suggestive of mycoplasma pneumonia; therefore, macrolide therapy is unnecessary. ${ }^{1}{ }^{2}$ The negative blood culture is not surprising given the low rate of bacteraemia associated with CAP $(1.4 \%-3.4 \%$ with uncomplicated and $7.8 \%$ with complicated CAP). International guidelines do not routinely recommend a blood culture in uncomplicated CAP. ${ }^{12}$

\section{SS AND AC}

After 48 hours of therapy, the patient remains persistently febrile, tachypnoeic, now with mild respiratory distress. Her systolic murmur is noted to be louder with no associated thrill. There are no peripheral stigmata of endocarditis. Three sets of blood cultures remain negative. Percussion and auscultation of the lung fields are unchanged, and her CRP has increased to $>270 \mathrm{mg} / \mathrm{L}$. A repeat CXR (figure 1B) shows dense consolidation of the left lower lobe, and of note, the area of radiolucency is no longer visible. There is also new right-sided retrocardiac opacification and tracheal deviation to the right. On review of the initial CXR, the tracheal deviation was present initially.

\section{AG (GENERAL PAEDIATRICIAN AND PAEDIATRIC INFECTIOUS DISEASES PHYSICIAN)}

In a patient with uncomplicated CAP, we would expect clinical improvement within 48 hours. ${ }^{12} \mathrm{~A}$ thorough clinical review, optimisation of antibiotic dosing and a repeat CXR are appropriate responses. There is no clinical evidence of pleural fluid, which would be the most common complication of bacterial pneumonia failing to respond to antibiotics. However, the CXR findings suggest progression of the infection despite appropriate antibiotic therapy. To delineate the presence of a pyogenic complication (ie, abscess) and the cause of tracheal deviation, a CT scan with contrast should be done. ${ }^{3}$ In the setting of a changing murmur, the previous echocardiogram result should be reviewed to ensure a structurally normal heart, and referral to cardiology considered.

\section{SS AND AC}

The CT scan shows extensive left lower lobe consolidation and multiple nodules throughout both lungs, some associated with ground-glass 
A

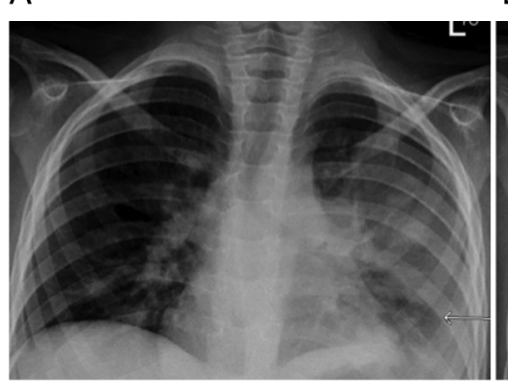

B

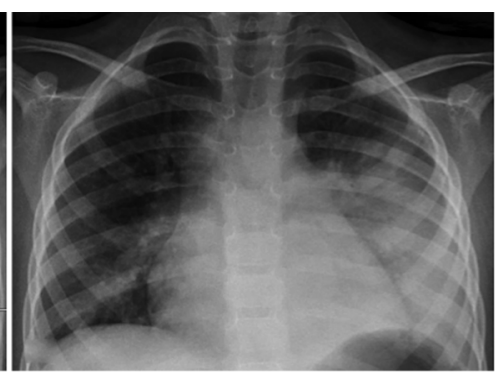

C

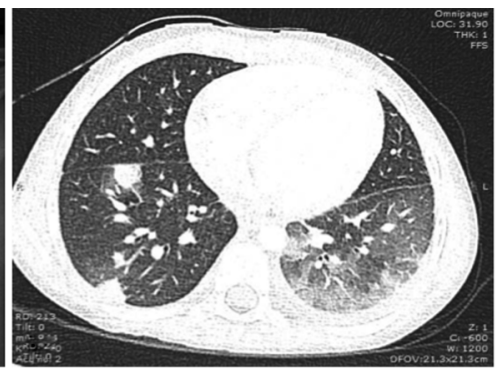

Figure 1 (A) A chest radiograph (CXR) showing left lower lobe consolidation with suggestion of abscess; (B). Repeat CXR showing worsening left lower lobe consolidation and tracheal deviation; (C). CT scan showing left lower lobe consolidation, bilateral lung nodules and some areas of ground-glass opacification.

opacification (figure 1C). In addition, there is bilateral hilar and mediastinal adenopathy and small bilateral pleural effusions. There is no evidence of an abscess. On review of the patient's cardiac history, the diagnosis of an innocent murmur was made on examination alone.

\section{AG}

The CT findings of widespread lung nodules raise a number of potential diagnoses. An infectious aetiology of the nodules is still most likely. One possibility is infective endocarditis given the risk of undiagnosed congenital heart disease, a changing murmur and persistent fever. In this setting, the nodules could represent septic emboli (secondary to right-sided endocarditis or in the setting of a left-to-right shunt). Pulmonary nodules with ground-glass opacification are suggestive of infection with an unusual organism such as fungi or atypical bacteria (ie, mycobacterial infection).

This patient has a low risk of colonisation with a resistant organism. ${ }^{4}$ Specifically, she has none of the risk factors (prior antibiotic use, indigenous ethnicity) which have been associated with paediatric methicillin-resistant Staphylococcus Aureus (MRSA) infections in Australia. ${ }^{5}$ She has no clinical features of Panton-Valentine leukocidin (PVL)-producing S. aureus necrotising pneumonia; therefore, empiric addition of antitoxin treatment such as clindamycin is not needed. Resistant Gram-negative organisms causing CAP is unlikely in children, even in those who do not respond to initial treatment. ${ }^{6}$ The patient's antibiotic regimen should be broadened to cover endocarditis (vancomycin and gentamicin), and treatment for complicated pneumonia (ceftriaxone) should be continued. Further investigation with a bronchoalveolar lavage (BAL) and echocardiography is indicated.

Other potential causes of lung nodules in children include malignancy and rheumatological disease causing pulmonary granulomas. The patient may need evaluation for these conditions if an infectious aetiology is not found.

\section{SS AND AC}

A transthoracic and transoesphageal echocardiogram show a structurally normal heart with no evidence of vegetations or valvular regurgitation. She remains febrile despite 5 days of intravenous antibiotics.

\section{JM (PAEDIATRIC RESPIRATORY PHYSICIAN) AND AG}

CT scans are rarely performed in paediatric CAP; so, the significance of the pulmonary nodules, ground-glass opacification and hilar adenopathy is unclear. Findings of pulmonary nodules have been described in CAP in adult patients, but is unusual and thought to represent either haematogenous spread of infection (classically varicella-zoster virus infection) or an alternate pathogen such as Mycobacterium tuberculosis, non-tuberculous mycobacteria or fungal infection causing granulomatous inflammation. ${ }^{7}$ Ground-glass opacification is more likely to be associated with infections due to viruses such as, Mycoplasma pneumoniae and Pneumocystis jirovecii. ${ }^{7}$ However, a halo of ground-glass attenuation around a pulmonary nodule could suggest infection with Aspergillus spp. ${ }^{8}$ The hilar adenopathy could be reactive to any pulmonary infection, but the constellation of findings (consolidation, nodules and lymphadenopathy) is suggestive of a more complex process than CAP.

\section{JM}

Of these potential infections, the most likely organism in an immunocompetent child is Mycoplasma pneumoniae, as it has been found to be a common cause of CAP that does not respond to standard therapy. ${ }^{6}$ Therefore, empiric treatment with a macrolide should be commenced. ${ }^{12}$

Given there has been no response to broad-spectrum antibiotics and the imaging findings suggest infection with an atypical pathogen, a BAL should be performed to identify atypical or resistant organisms. A recent retrospective review found that in children with CAP that did not respond to initial antibiotic therapy, a BAL resulted in identification of a pathogen in $75 \%{ }^{6}$ This high yield is potentially explained by the use of novel diagnostic techniques (ie, molecular microbiological tests such as PCR). The BAL sample should be tested for typical (microscopy, culture and susceptibilities) and atypical organisms (atypical organism PCR), mycobacteria (Ziehl-Neelsen stain, mycobacterial culture and pan-mycobacterial PCR) and fungal pathogens (wet preparation, fungal culture and $18 \mathrm{~S}$ ribosomal RNA PCR).

\section{MM (GENERAL PAEDIATRICIAN)}

This patient has not improved clinically despite appropriate antibiotic therapy, and her imaging suggests an unusual disease process. The bronchoscopic examination of her airways is normal, and no organism has been isolated from lavage. She has a history of three episodes of otitis media, resulting in tympanic membrane perforation. She should therefore be investigated for an immune deficiency. Although she does not fulfil the criteria for the commonly used ' 10 warning signs for primary immune deficiency', these criteria must be interpreted with caution, as they have not been validated in paediatric clinical studies. ${ }^{9}$ A recent study identified risk factors such as family history, sepsis requiring intravenous antibiotics and failure to thrive as the 
most specific markers of an immune disorder in a British population. ${ }^{9}$

\section{TC (PAEDIATRIC IMMUNOLOGIST)}

This clinical presentation and lack of response to initial therapy warrants referral to an immunologist for investigation for an underlying immunodeficiency. With a history of sinopulmonary infections, it is important to consider an antibody defect. However, the CT findings of multiple lung nodules with hilar lymphadenopathy could be consistent with fungal infection in the context of chronic granulomatous disease, and a nitroblue tetrazolium and/or dihydrorhodamine assay should be done. Given the possibility of a fungal infection, investigations for defects in T cell function and an HIV test should be performed.

\section{SS AND AC}

Immune function testing is performed. Her full blood examination, lymphocyte subsets, immunoglobulins, naïve $\mathrm{T}$ cells, memory B cells and complement studies were normal. The neutrophil oxidative burst is absent on both nitroblue tetrazolium and dihydrorhodamine assay testing.

\section{TC (PAEDIATRIC IMMUNOLOGIST)}

Her neutrophil function test result is consistent with a diagnosis of chronic granulomatous disease (CGD). Given the patient is female, this is most likely an autosomal recessive form. Patients with CGD are predisposed to infection with catalase-positive organisms, including staphylococci, Burkholderia cepacia, Serratia spp., Nocardia spp. and Aspergillus spp. Given the high risk of invasive fungal infection, this patient should be commenced on voriconazole for empiric treatment of invasive pulmonary aspergillosis. In addition, a galactomannan test should be added to the BAL sample retrospectively, and a radiologically guided biopsy of one of the pulmonary nodules should be considered. CT screening for other sites of infection should also be done.

\section{SS AND TC}

No pathogens were identified on culture from radiologically guided biopsy of the area of consolidation. The BAL galactomannan was positive $(1.25,>0.5$ abnormal) suggesting a probable diagnosis of invasive pulmonary aspergillosis. Identification of invasive fungal infection in patients with CGD can be difficult. A review of patients with CGD on the French national registry demonstrated that surgical biopsy was required in $86 \%$ of cases to identify invasive mould infection, with culturepositive results of only $21 \%$ and $28 \%$ for needle aspiration and BAL, respectively. ${ }^{10}$ The patient was treated with voriconazole, with trough levels of $0.24-5.82 \mathrm{mg} / \mathrm{L}$. Despite this, the patient remained febrile, and adjunctive corticosteroids were commenced to reduce the inflammatory response with good effect. Further testing confirmed p47-phox deficiency which is a component of nicotinamide adenine dinucleotide phosphate (NADPH) oxidase, a known cause of autosomal recessive CGD. ${ }^{11}$ The patient is currently being evaluated for a haemopoietic stem cell transplant.

\section{SS AND AG}

This is a classic presentation of autosomal recessive CGD. A multicentre retrospective review of 60 patients with autosomal recessive CGD reported an average age of diagnosis of 5.8 years, with pulmonary infection being the most common initial presentation. ${ }^{12}$ This differs from X-linked CGD where symptoms often develop in the first year of life and the diagnosis is frequently made before the age of 5 years.

This case highlights a stepwise approach to the management of patients who do not respond to standard therapy for CAP. Current international guidelines recommend empiric antibiotic treatment and watchful waiting for the first 48 hours; however, there are no published guidelines on how to investigate and manage cases that do not respond within this time frame. ${ }^{1}{ }^{2}$ In this scenario, the following should always be considered:

1. Are secondary pyogenic complications present? (ie, empyema, lung abscess);

2. Could this be an infection caused by a resistant organism? (this largely depends on local resistance patterns);

3. Could this infection be due to an alternative pathogen? (ie, viral, atypical or fungal pathogen);

4. Is there an alternative primary site of infection? (ie, infective endocarditis, Lemierre syndrome);

5. Is this patient a normal host? (ie, presence of an underlying predisposing condition such as non-infectious aetiology or first presentation of immune deficiency).

The likelihood and need to investigate for the above differentials will vary depending on the patient's history and presentation; therefore, an individualised approach for each case is needed.

Contributors All authors contributed to the care of the patient and the completion of the manuscript.

Competing interests None declared.

Patient consent Parental/guardian consent obtained.

Provenance and peer review Not commissioned; externally peer reviewed.

\section{REFERENCES}

1 Harris M, Clark J, Coote N, et al. British Thoracic Society guidelines for the management of community acquired pneumonia in children: update 2011. Thorax 2011;66(Suppl 2):ii1-23.

2 Bradley JS, Byington CL, Shah SS, et al. The management of community-acquired pneumonia in infants and children older than 3 months of age: clinical practice guidelines by the Pediatric Infectious Diseases Society and the Infectious Diseases Society of America. Clin Infect Dis 2011;53:e25-76.

3 Patradoon-Ho P, Fitzgerald DA. Lung abscess in children. Paediatr Respir Rev 2007:8:77-84.

4 Shindo $Y$, Ito R, Kobayashi D, et al. Risk factors for drug-resistant pathogens in community-acquired and healthcare-associated pneumonia. Am J Respir Crit Care Med 2013;188:985-95.

5 Britton PN, Andresen DN. Paediatric community-associated Staphylococcus aureus: a retrospective cohort study. J Paediatr Child Health 2013;49:754-9.

6 De Schutter I, De Wachter E, Crokaert F, et al. Microbiology of bronchoalveolar lavage fluid in children with acute nonresponding or recurrent community-acquired pneumonia: identification of nontypeable Haemophilus influenzae as a major pathogen. Clin Infect Dis 2011;52:1437-44.

7 Nambu A, Ozawa K, Kobayashi N, et al. Imaging of community-acquired pneumonia: roles of imaging examinations, imaging diagnosis of specific pathogens and discrimination from noninfectious diseases. World J Radiol 2014;6:779-93.

8 Westra SJ, Brody AS, Mahani MG, et al. The incidental pulmonary nodule in a child. Part 1: recommendations from the SPR Thoracic Imaging Committee regarding characterization, significance and follow-up. Pediatr Radiol 2015;45:628-33.

9 Subbarayan A, Colarusso G, Hughes SM, et al. Clinical features that identify children with primary immunodeficiency diseases. Pediatrics 2011;127:810-16.

10 Blumental S, Mouy R, Mahlaoui N, et al. Invasive mold infections in chronic granulomatous disease: a 25-year retrospective survey. Clin Infect Dis 2011;53: e159-69.

11 Roos D, de Boer M, Kuribayashi F, et al. Mutations in the X-linked and autosomal recessive forms of chronic granulomatous disease. Blood 1996;87:1663-81.

12 Martire B, Rondelli R, Soresina A, et al. Clinical features, long-term follow-up and outcome of a large cohort of patients with Chronic Granulomatous Disease: an Italian multicenter study. Clin Immunol 2008;126:155-64. 